\title{
Influencia del Volumen e Intensidad de la Carga de Entrenamiento en la Frecuencia Cardiaca de Recuperación \\ Influence of Training Load Volume and Intensity on Heart Rate Recovery \\ *Roberto Andrés González-Fimbres, *Héctor Griego Amaya, *Claudia SeleneCuevas-Castro, **Germán Hernández Cruz *Universidad Estatal de Sonora (México), **Universidad Autónoma de Nuevo León
}

Resumen. Cuantificar los componentes de volumen e intensidad de la carga de entrenamiento es importante para garantizar la mejora del rendimiento. El objetivo de este estudio fue el comparar los efectos del volumen y la intensidad de la carga de entrenamiento sobre la Frecuencia Cardiaca de Recuperación (FCR). Dos mujeres y cuatro hombres, (edad M: $21 \pm 1.41$, H: $25.75 \pm 4.57$ años) entrenados en deportes de resistencia llevaron a cabo dos tratamientos con la misma carga interna (TRIMP $=52$ unidades arbitrarias) pero con variación en volumen e intensidad (T1 = intensidad alta y volumen bajo [86-91\% FCres, 14.5 min, T2 = intensidad baja y volumen alto [72-78\% FCres, 30.5 min]). Posterior al ejercicio se monitoreó la FCR en cinco momentos: al finalizar el esfuerzo (R1), 10 (R2), 20 (R3), 30 (R4) y 40 (R5) minutos después del esfuerzo. Se encontraron diferencias significativas ( $p \mathrm{~d}+.05)$ entre los valores de FCR en los cinco momentos. Los resultados sugieren que tratamientos de la misma carga interna existen diferencias entre la carga interna de los tratamientos, observando que la intensidad afecta de mayor manera a la FCR que el volumen. Palabras Clave. TRIMP, Frecuencia Cardiaca, evaluación, ejercicio.

Abstract. Quantifing the effects of volume and intensity components of training load (TL) is essential in order to guarantee performance enhancement. The aim of this study was to compare the effects of training load volume and intensity on Heart Rate Recovery (HRR). Two women (age $=21 \pm 1.41$ ) and four men (age $=25.75 \pm 4.57$ years) trained in endurance sports performed two different treatments with equal TL (TRIMP $=$ 52 arbitrary units) but different volume and intensity (Training 1 = high intensity, low volume [86-91\% HRres, 14.5 min], Training 2 = low intensity, high volume [72-78\% HRres, 30.5 min]). HRR was monitored after exercise in five moments: at the end of training (R1), and 10 (R2), 20 (R3), 30 (R4), and 40 (R5) minutes after effort. Significant differences were found in HRR values at each of the five intervals ( $p$ d+ .05). Results suggest the existence of significant differences in TL between the two treatments. Outcomes also evidenced that intensity has a greater effect than volume on HRR.

Key words. TRIMP, Heart Rate, evaluation, exercise.

\section{Introducción}

En la preparación deportiva existen dos formas de considerar la cuantificación de la carga de entrenamiento: interna y externa. La carga externa es el trabajo realizado por el atleta medido independientemente de sus efectos internos. La carga interna es el estrés fisiológico y psicológico impuesto al atleta durante el entrenamiento (Foster et al., 2001; Halson, 2014; Impellizerrri, Rampini \& Marcora, 2005). Controlar la carga interna resulta fundamental para asegurar que el entrenamiento realizado provoque las adaptaciones fisiológicas deseadas y evitar un posible sobre-entrenamiento (Le Meur, Hausswith, Natta, Couturier, Bignet \& Vidal, 2013).

Para cuantificar la carga interna de entrenamiento se deben de considerar los elementos que la componen: volumen, intensidad y densidad (Hartmann, Bob, Wirth \& Schmidtbleicher, 2009). Existen estudios que han analizado la distribución del volumen e intensidad de la carga de entrenamiento en deportes de resistencia, y los efectos que esta distribución tiene en el desempeño del deporte (Billat, Lepretre, Heugas, Laurence, Salim \& Koralsztein, 2003; Esteve-Lanao, San Juan, Earnest, Foster \& Lucia, 2005; Seiler \& Kjerland, 2006). Se considera que la intensidad del ejercicio es un elemento clave en un programa de entrenamiento para el desarrollo de la capacidad aeróbica, aunque todavía existen controversias sobre cuál es la intensidad adecuada para provocar el estímulo necesario para que se lleven a cabo las adaptaciones fisiológicas (Carazo-Vargas \& Moncada-Jiménez, 2015).

La cuantificación de la carga representa dificultades para los entrenadores, ya que la recolección, control e interpretación de los resultados requieren de un alto grado de capacitación y el uso de equipamiento sofisticado para hacer las mediciones (Halson, 2014). Por otra parte, métodos de cuantificación de la carga que involucren marcadores biológicos en sangre tienen carácter invasivo y costoso, además del inconveniente sobre el manejo de las muestras y el requerimiento de personal especializado (Bocanegra, Diaz, Teixeira, Soares \& Espindola, 2012). Uno de los métodos que se ha estudiado recientemente para medir la

Fecha recepción: 05-01-16. Fecha de aceptación: 20-05-16 M.A. Roberto Andrés González-Fimbres roberto.led.ues@gmail.com carga interna por medio de la frecuencia cardiaca (FC) es el TRIMP (Training Impulse) propuesto por Banister (1991) como un método para cuantificar la carga interna de entrenamiento (García-Ramos et al., 2015; Manzi, Bovenzi, Impellizzeri, Carminati \& Castgna, 2013; Scott, Lockie, Knight, Clark \& Janse de Jonge, 2013; Wallace,

Slattery \& Coutts, 2014). El TRIMP toma en consideración la intensidad del ejercicio al calcularla por medio del método de la FC de reserva (FCres) y el volumen por medio de la duración en minutos del ejercicio. El promedio de la FC por la sesión de entrenamiento es ponderado de acuerdo a la relación entre FC y el lactato sanguíneo

(LS), al observarse durante el incremento del ejercicio y luego se multiplica por la duración de la sesión (Banister, 1991).

Una de las limitantes del TRIMP de Banister es que el aumento de la capacidad de rendimiento de los atletas provoca un desplazamiento hacia la derecha en la gráfica de producción de LS durante la ejecución de una prueba incremental. La ecuación que pondera el LS no considera el estado del atleta por lo que los resultados no son consistentes con personas de diferentes niveles de condición física (Faude, Kindermann \& Meyer, 2009).

Para atender esta limitante Lucia et al. (2003) propusieron un método de cuantificación del TRIMP multiplicando la duración del ejercicio por un coeficiente de ponderación $(1,2$ y 3 ) de acuerdo al tiempo que hayan transcurrido en tres zonas definidas por umbrales ventilatorios, zona I ( $<70 \% \mathrm{VO}_{2}$ max), zona II (entre el $70 \%$ y el $90 \% \mathrm{VO}_{2}$ max), y zona III ( $>90 \%$ del $\mathrm{VO}_{2}$ max $)$.

Seiler \& Kjerland (2006) propusieron clasificar la FC en tres zonas de entrenamiento en base a la producción de LS en lugar de los umbrales ventilatorios, la primer zona de entrenamiento de bajo lactato $<2 \mathrm{mmol} /$ l, la segunda zona de acomodación de lactato entre $2 \mathrm{mmol} / \mathrm{l}$ y $4 \mathrm{mmol} /$ l, la tercer zona de acumulación de lactato $>4 \mathrm{mmo} / \mathrm{l}$. Sin embargo, no atendieron la limitación de justificar fisiológicamente los factores de ponderación de cada zona.

Para justificar los factores de ponderación de las zonas de entrenamiento, Stagno, Thatcher \& Van Someren (2007) desarrollaron una versión modificada del TRIMP de Banister, midieron directamente el perfil de LS de jugadores de hockey. Por lo tanto, las ponderaciones que utilizaron reflejan el perfil de una curva de respuesta de lactato típica al aumento de las intensidades del ejercicio, estableciendo cinco zonas de 
FC basadas en los umbrales de LS y el inicio de la acumulación del lactato sanguíneo (Onset of Blood Lactate Accumulation, OBLA), siendo los resultados de las zonas de ponderación: 1.25, 1.71, 2.54, 3.61 y 5.16. El tiempo acumulado en cada zona de FC era entonces multiplicado por su respectivo factor de ponderación para derivar un TRIMP modificadogeneral.

Otro método de cuantificación de la carga interna de entrenamiento por medio de la FC es la frecuencia cardiaca de recuperación (FCR), entendida como el ritmo en el cual la FC disminuye una vez terminado el ejercicio. Daanen, et al. (2012) mencionan la FCR como un marcador de la función del Sistema Nervioso Autónomo(SNA), el cual comprende los sistemas simpático y parasimpático. La FCR se caracteriza por un aumento de la actividad parasimpática y un retiro de la actividad simpática (Halson, 2014).

Borressen \& Lambert (2007) encontraron en su estudio que sujetos entrenados mantienen sus valores de FCR al sostenerse las cargas de entrenamiento, la recuperación cardiaca es más lenta si se aumentan las cargas de entrenamiento, y muestran una recuperación más rápida si se disminuyen las cargas de entrenamiento, por lo que sugieren la utilización de la FCR como un marcador de la carga de entrenamiento.

Seiler, Haugen \& Kuffel (2007) encontraron que a mayor intensidad del ejercicio resultan mayores valores de FC y una rápida disminución de la FCR en los primeros minutos. Por el contrario, otros estudios sostienen que después de ejercicios máximos la FCR tiende a ser más lenta, debido a que la activación simpática puede prolongarse durante las primeras etapas de recuperación (Kaikkonen, et al., 2008; Lamberts \& Lambert, 2009). Por otra parte, la duración del ejercicio tiene menor efecto sobre la FCR que la intensidad (Seiler, et al., 2007).

Se ha observado que la FCR aumenta (los atletas se recuperan más rápido) después de un tratamiento de entrenamiento de alta intensidad, además que los sujetos que incrementaban constantemente su FCR tenían mejor desempeño en una prueba de $40 \mathrm{~km}$ en ciclistas elite (Lamberts, Swart, Capostagno, Noakes \& Lambert, 2010), por lo que la FCR también puede ser un predictor del desempeño en deportes de carga continua. En ejercicios de intensidad continua de larga duración se ha observado una FCR más alta en ejercicios que tienen mayor demanda aeróbica (Maeder, Ammann, Rickli, Brunner-La Rocca, 2009), es por ello que el tipo de ejercicio ejecutado afecta los valores de FCR.

Rusko et al. (2004) llevaron a cabo un estudio donde compararon el efecto de la manipulación del volumen y la intensidad en ejercicios con el mismo gasto calórico en la carga interna evaluada por medio del Exceso de Consumo de Oxígeno Post Ejercicio (Excess Postexercise Oxygen Consumption, EPOC) y el TRIMP. Se encontró que con el aumento de la duración del ejercicio se indujeron mayores cambios en el TRIMP, mientras que el aumento de la intensidad mostraba mayores cambios en el Índice de Esfuerzo Percibido (Ratio of Perceived Exertion, RPE), LS, FC y EPOC.

El estudio de Rusko et al. (2004) no consideró la relación que existiría entre ejercicios con el mismo nivel del TRIMP con los efectos post ejercicio, por lo que el objetivo del presente estudio fue el comparar los efectos del volumen y la intensidad de la carga de entrenamiento sobre la Frecuencia Cardiaca de Recuperación(FCR)

\section{Metodología}

\section{Participantes}

Se seleccionaron por conveniencia a seis sujetos, cuatro hombres y dos mujeres, con las siguientes características (media \pm DS): Hombres: edad $25.75 \pm 4.57$ años, masa corporal $71.9 \pm 9.94$ kg., estatura $173 \pm$ $6.92 \mathrm{~cm}$; Mujeres: edad $21 \pm 1.41$ años, masa corporal $51.45 \pm 1.34 \mathrm{~kg}$., estatura $159 \pm 4.24 \mathrm{~cm}$. Los sujetos fueron atletas entrenados que participaban en pruebas de fondo y triatlón, contaban por lo menos con tres años de experiencia en la práctica deportiva. Se les aplicó un historial médico para descartar alguna enfermedad, consumo de medicamento o suplemento, ausencia de lesión que afectara el desempeño en la prueba, número de horas de sueño previas. Todos los sujetos participaron de forma voluntaria y firmaron un consentimiento informado.

\section{Instrumentos}

Para el monitoreo de la FC se utilizaron Pulsómetros Polar RCX5 (Finlandia) con interface de exportación de datos a computadora para posterior manejo y análisis. Los tratamientos de ejercicios se llevaron a cabo en un tapiz rodante Freemotion (E.U.) con motor de 4.25 CHP con velocidad máxima de 12 mph compatible con Pulsómetro Polar.

\section{Procedimiento}

Se solicitó a los sujetos una semana previa de iniciar el estudio suspender su entrenamiento regular. Al momento de la aplicación de los tratamientos se les pidió ayuno de por lo menos dos horas.

La FC de reserva (FCres) de los sujetos se calculó restando la FC de reposo a la FC máxima (FCmax). La frecuencia cardiaca en reposo se determinó colocando al sujeto en posición supina por un periodo de 30 minutos, considerando el valor mínimo registrado en ese periodo. La FCmax se obtuvo registrando el valor máximo de FC observado en una prueba de esfuerzo incremental en tapiz rodante hasta el agotamiento voluntario. La velocidad inicial se estableció a $8 \mathrm{~km} / \mathrm{h}$ en el primer minuto, al segundo minuto se incrementa a $10 \mathrm{~km} / \mathrm{h}$, posteriormente se incrementa $0.5 \mathrm{~km} / \mathrm{h}$ cada 30 segundos.

Se sometió a los sujetos a dos tratamientos de cargas de esfuerzo con el mismo TRIMP (52 unidades arbitrarias) según la metodología de Stagno et al. (2007). El primer tratamiento consistió en ejercicio de alta intensidad (zona 4, 86-91\% FCres) y volumen de 14.5 min. Una semana después se llevó a cabo el segundo tratamiento de baja intensidad (zona 2, 72-78\% FCres) y volumen de $30.5 \mathrm{~min}$. Todos los tratamientos fueron aplicados a la misma hora con temperatura ambiente constante de $24^{\circ} \mathrm{C}$. La intensidad del ejercicio estuvo regulada por la velocidad del tapiz rodante.

Al finalizar cada tratamiento los sujetos se colocaron en posición supina para su recuperación, se monitoreó la FCR haciendo registros justo al finalizar el esfuerzo (R1), a los 10 (R2), 20 (R3), 30 (R4) y 40 (R5) minutos después del esfuerzo.

Los datos de FC absoluta del esfuerzo (FCE) arrojados por los sujetos fueron convertidos a porcentaje de la FCres por medio de la fórmula:

$$
\% \text { FCres }=\left(\frac{F C E-F C R}{F C \max -F C R}\right) \times 100
$$

El registro de FC en cada momento (R1-R5) fue la media de la FCres del último minuto del periodo monitoreado.

\section{Análisis estadístico de los datos}

Para el análisis de los datos se utilizó el software IBM SPSS Statistics Versión 22 (E.U.). Todos los datos se expresan como media \pm desviación estándar. La normalidad de los datos se realizó a través del test de Shapiro-Wilk, posteriormente se llevó a cabo una prueba $t$ student para pruebas relacionadas para determinar diferencias entre los dos tratamientos del mismo grupo. El nivel de significancia se estableció en $p \mathrm{~d} » .05$

\section{Resultados}

\section{Descripción inicial de los datos}

Los resultados descriptivos individuales y del grupo se presentan en la Tabla 1, contrastando los valores de porcentaje de FCres obtenidos en los dos tratamientos en cada uno de los momentos de recuperación.

La figura 1 muestra las tendencias de la frecuencia cardica del tratamiento uno (intensidad baja, volumen alto) y el tratamiento dos (intensidad alta, volumen bajo).

En ambos tratamientos se observa una disminución de la FC en cada uno de los momentos del periodo de recuperación (R1-R5). En ninguno de los dos tratamientos los sujetos lograron llegar a los niveles basales de FC después de 40 minutos de recuperación, por lo que se considera que en ninguno de los casos no se llegó una recuperación completa.

\section{Análisis}

Los resultados de la comparación entre los dos tratamientos se 
Tabla 1.

Porcentaje de la FCres en los distintos momentos de recuperación

\begin{tabular}{ccccccc}
\hline \multirow{2}{*}{ Sujeto } & \multirow{2}{*}{ Tratamiento } & \multicolumn{5}{c}{ \% FCres } \\
\cline { 2 - 7 } & & R1 & R2 & R3 & R4 & R5 \\
\hline \multirow{2}{*}{1} & T1 & 54.78 & 17.45 & 13.42 & 11.40 & 3.98 \\
& T2 & 73.34 & 27.76 & 17.34 & 12.87 & 6.98 \\
\hline \multirow{2}{*}{2} & T1 & 43.60 & 10.23 & 6.44 & 4.82 & 4.33 \\
& T2 & 67.48 & 25.67 & 20.85 & 14.89 & 15.43 \\
\hline \multirow{2}{*}{3} & T1 & 47.63 & 16.96 & 11.89 & 9.76 & 7.47 \\
& T2 & 57.80 & 27.93 & 24.49 & 17.89 & 14.67 \\
\hline \multirow{2}{*}{4} & T1 & 56.15 & 15.10 & 14.66 & 11.65 & 9.54 \\
& T2 & 67.99 & 34.16 & 30.43 & 25.75 & 20.06 \\
\hline \multirow{2}{*}{5} & T1 & 63.44 & 20.82 & 16.71 & 14.05 & 12.00 \\
& T2 & 71.33 & 34.25 & 21.33 & 16.51 & 14.66 \\
\hline \multirow{2}{*}{6} & T1 & 47.56 & 15.64 & 12.86 & 12.12 & 10.04 \\
& T2 & 78.93 & 31.56 & 25.58 & 19.97 & 16.87 \\
\hline \multirow{2}{*}{ Grupo } & T1 & $\mathbf{5 2 . 2 0} \pm 7.28$ & $\mathbf{1 6 . 0 3} \pm \mathbf{3 . 4 8}$ & $\mathbf{1 2 . 6 6} \pm \mathbf{3 . 4 7}$ & $\mathbf{1 0 . 6 3} \pm \mathbf{3 . 1 6}$ & $\mathbf{7 . 8 9} \pm \mathbf{3 . 2 4}$ \\
& T2 & $\mathbf{6 9 . 4 8} \pm \mathbf{7 . 0 7}$ & $\mathbf{3 0 . 2 2} \pm \mathbf{3 . 6 2}$ & $\mathbf{2 3 . 3 4} \mathbf{\pm 4 . 5 3}$ & $\mathbf{1 7 . 9 8} \pm \mathbf{4 . 5 2}$ & $\mathbf{1 4 . 7 8} \mathbf{\pm 4 . 3 3}$ \\
\hline
\end{tabular}

$\begin{array}{llllll}\text { \% FCres = Porcentaje de la frecuencia cardiaca de reserva, R1 } & \text { R m momento justo después del }\end{array}$ esfuerzo, R2 $=10$ min posterior al esfuerzo, R3 $=20$ min posterior al esfuerzo, R4 $=30 \mathrm{~min}$ posteior intensidad baja y volumen alto, T2 se refiere al tratamiento dos de intensidad alta y volumen bajo.

Tabla 2 .

Resultados de la prueba $t$-student para muestras relacionadas entre los dos tratamientos.

\begin{tabular}{|c|c|c|c|c|}
\hline \multirow{2}{*}{\multicolumn{2}{|c|}{ Momento }} & \multicolumn{2}{|c|}{ Medias \%FCres } & \multirow{2}{*}{$\begin{array}{c}\text { Nivel de } \\
\text { significan } \\
\text { cia } p\end{array}$} \\
\hline & & T1 & T2 & \\
\hline Par 1 & R1 & $52.20 \pm 7.28$ & $69.48 \pm 7.07$ & $.006^{*}$ \\
\hline Par 2 & R2 & $16.03 \pm 3.48$ & $30.22 \pm 3.62$ & $.000^{*}$ \\
\hline Par 3 & R3 & $12.66 \pm 3.47$ & $23.34 \pm 4.53$ & $.004 *$ \\
\hline Par 4 & R4 & $10.63 \pm 3.16$ & $17.98 \pm 4.52$ & $.013^{*}$ \\
\hline Par 5 & R5 & $7.89 \pm 3.24$ & $14.78 \pm 4.33$ & $.042^{*}$ \\
\hline
\end{tabular}

$\mathrm{y}$ volumen alto, $\mathrm{T} 2=$ tratamiento dos de intensidad alta y volumen bajo, $*$ indica que existen diferencias significativas entre los pares de tratamiento $p=.05$.

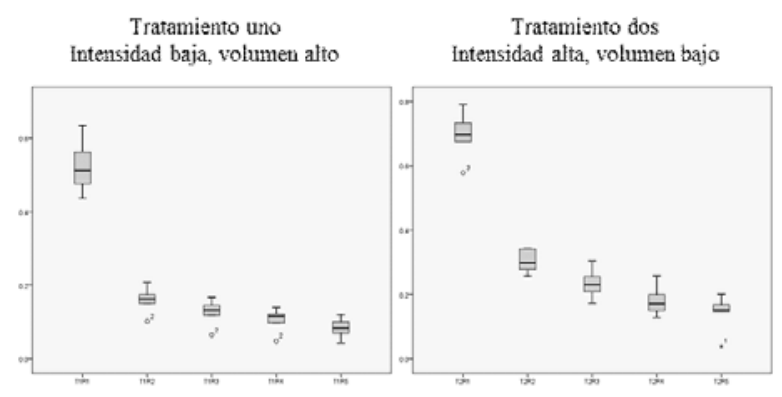

Figura 1. Dinámica de la frecuencia cardiaca de reserva del tratamiento uno (Intensidad baja, volumen alto) y tratamiento dos (Intensidad alta, volumen bajo).

presentan en la tabla 2, observando diferencias significativas ( $p>.05)$ entre cada uno de los momentos del periodo de recuperación. Las mayores diferencias se observan entre los registros R1 y R2.

Al acercarce la FCR a los valores basales, comienza a disminuir la significancia de la prueba $t$, sin embargo, hasta el último momento registrado (R5, 40 min posterior) la diferencia sigue siendo significativa.

La recuperación entre los dos tratamientos muestra diferentes patrones, siendo más retrasada la del tratamiento de alta intensidad, lo que sugiere que representó una mayor carga interna en los participantes, aunque la cuantificación del TRIMP haya sido la misma.

\section{Discusión}

El objetivo de este estudio fue comparar los efectos del volumen y la intensidad de la carga de entrenamiento sobre la FCR. El resultado principal de este estudio fue observar que el tratamiento de alta intensidad y baja duración tuvo una FCR más lenta que el tratamiento de baja intensidad y alta duración, a un mismo valor de carga interna de entrenamiento medida por el TRIMP.

Se pudo observar que aunque los valores de TRIMP en ambos tratamientos eran iguales, los valores de FCR en todos los momentos (R1-R5) tenían diferencias significativas. Esto contradice lo mencionado por Lamberts et al. (2010) y Borresen \& Lambert (2007), quienes sostienen que la FCR aumenta cuando hay un aumento en la carga de entrenamiento y esta se mantiene constante si la carga de entrenamiento es constante. Esta contradicción sugiere que la FCR es un valor independiente del TRIMP. Una carga de entrenamiento con componente de intensidad alto aparenta provocar un mayor estrés fisiológico post- ejercicio que una carga de magnitud similar con componente de intensidad bajo. Aparentemente el TRIMP es una medición del estrés durante el ejercicio y la FCR es un marcador del estrés post-ejercicio.

Los valores de FC más elevados y el mayor ritmo de FCR observados en el tratamiento de alta intensidad son consistentes con el estudio de Seiler et al. (2007), quienes sostienen que entre mayor sea la intensidad del ejercicio, mayor será la FC alcanzada y una más rápida FCR. Cuando se realiza un ejercicio cercano al nivel máximo de carga se puede observar una desactivación retrasada el sistema simpático, lo que provoca que la FCR sea más lenta (Kaikkonen et al., 2008; Lamberts \& Lambert, 2009), lo que también se comprueba en los datos observados del estudio, al ser más lenta la FCR en el tratamiento de alta intensidad comparada con la del tratamiento de baja intensidad en los minutos posteriores.

Los datos observados sugieren validar los resultados de Seiler et al. (2007), los cuales indican que una variación en la intensidad tiene mayor efecto en la FCR que la duración del ejercicio.

La mayoría de los estudios encontrados utilizan un tiempo de FCR con un rango entre 30 segundos y un minuto. Solamente el estudio de Seiler et al. (2007) reporta haber monitoreado la FC por un periodo de hasta 240 minutos, donde no encontraron diferencias significativas entre los valores de FCR entre ejercicios de 60 y 120 minutos de baja intensidad.

Dentro de las limitaciones de este estudio, consideramos que es necesario continuar investigando las formas de cuantificación de la carga por medio del TRIMP, tomando en cuenta los umbrales de producción de LS individual en base a los resultados obtenidos en una prueba de esfuerzo incremental. Se considera conveniente relacionar otras variables con la manipulación del volumen y la intensidad del ejercicio adoptando otros diseños de experimentación, contrastando valores de $\mathrm{VO}_{2}$ max, $\mathrm{VO}_{2}$, EPOC y variabilidad de la FC además de los valores de TRIMP y FCR.

\section{Conclusiones}

Se observó que sesiones de ejercicio con el mismo TRIMP (52 unidades arbitrarias) que tenían diferentes valores de volumen e intensidad arrojaron diferencias significativas en los valores de FCR, lo que sugiere que el método de TRIMP subestima el aporte de la intensidad del ejercicio a la carga total de entrenamiento.

Estos resultados permiten a los entrenadores dosificar las cargas de entrenamiento brindando mayor tiempo de recuperación cuando se realicen ejercicios de mayor intensidad.

\section{Referencias}

Banister, E.W., MacDougall, J. D., Wenger, H. A., \& Green, H. J. (Eds.). (1991). Physiological testing of the high-performance athlete. Champaign, Ill.: Human Kinetics Books, 403-25.

Billat, V., Lepretre, P. M., Heugas, A. M., Laurence, M. H., Salim, D., \& Koralsztein, J.P. (2003). Training and bioenergetic characteristics in elite male and female Kenyan runners. Medicine and Science in Sports and Exercise, 35(2), 297-304.

Bocanegra, O. L., Diaz, M. M., Teixeira, R. R., Soares, S. S., \& Espindola, F. S. (2012). Determination of the lactate threshold by means of salivary biomarkers: chromogranin A as novel marker of exercise intensity. European journal of applied physiology, 112(9), 31953203.

Borresen, J., \& Lambert, M. I. (2007). Changes in heart rate recovery in response to acute changes in training load. European journal of applied physiology, 101(4), 503-511.

Daanen, H. A., Lamberts, R. P., Kallen, V. L., Jin, A., \& Van Meeteren, N. L. (2012). A systematic review on heart-rate recovery to monitor changes in training status in athletes. International Journal of Sports Physiology and Performance. 7(3), 251-260.

Esteve-Lanao, J., San Juan, A.F., Earnest, C.P., Foster, C., \& Lucia, A. (2005) How do endurance runners actually train? Relationship 
with competition performance. Medicine \& Science in Sports \& Exercise, 37(3), 496-504.

Faude, O., Kindermann, W., \& Meyer, T. (2009). Lactate threshold concepts. Sports medicine, 39(6), 469-490.

Foster, C., Florhaug, J. A., Franklin, J., Gottschall, L., Hrovatin, L. A., Parker, S.,... \& Dodge, C. (2001). A new approach to monitoring exercise training. The Journal of Strength \& Conditioning Research, 15(1), 109-115.

García-Ramos, A., Feriche, B., Calderón, C., Iglesias, X., Barrero, A., Chaverri, D.,... \& Rodríguez, F. A. (2015). Training load quantification in elite swimmers using a modified version of the training impulse method. European Journal of Sport Science, 15(2), 85-93.

Halson, S. L. (2014). Monitoring training load to understand fatigue in athletes. Sports Medicine, 44(2), 139-147.

Hartmann, H., Bob,A., Wirth, K., \& Schmidtbleicher, D. (2009). Effects of different periodization models on rate of force development and power ability of the upper extremity. The Journal of Strength \& Conditioning Research, 23(7), 1921-1932.

Impellizzeri, F. M., Rampinini, E., \& Marcora, S. M. (2005). Physiological assessment of aerobic training in soccer. Journal of Sports Sciences, 23(6), 583-592.

Kaikkonen, P., Rusko, H., \& Martinmäki, K. (2008). Post exercise heart rate variability of endurance athletes after different high intensity exercise interventions. Scandinavian Journal of Medicine \& Science in Sports, 18(4), 511-519.

Carazo-Vargas, P., \& Moncada-Jiménez, J. (2015). A meta-analysis on the effects of exercise training on the VO2max in children and adolescents (Meta análisis de los efectos del entrenamiento en el VO2máx en niños y adolescentes). Retos, (27), 184-187.

Lamberts, R. P., \& Lambert, M. I. (2009). Day-to-day variation in heart rate at different levels of submaximal exertion: implications for monitoring training. The Journal of Strength \& Conditioning Research, 23(3), 1005-1010.

Lamberts, R. P., Swart, J., Capostagno, B., Noakes, T. D., \& Lambert, M. I. (2010). Heart rate recovery as a guide to monitor fatigue and predict changes in performance parameters. Scandinavian Journal of Medicine \& Science in Sports, 20(3), 449-457.

Le Meur, Y., Hausswirth, C., Natta, F., Couturier, A., Bignet, F., \& Vidal, P. P. (2013). A multidisciplinary approach to overreaching detection in endurance trained athletes. Journal of Applied Physiology, 114(3), 411-420.

Lucia, A., Hoyos, J., Santalla, A., Earnest, C., \& Chicharro, J. L. (2003). Tour de France versus Vuelta a Espana: which is harder?. Medicine and Science in Sports and Exercise, 35(5), 872-878.

Maeder, M. T., Ammann, P., Rickli, H., \& Brunner-La Rocca, H. P. (2009). Impact of the exercise mode on heart rate recovery after maximal exercise. European journal of applied physiology, 105(2), 247-255.

Manzi, V., Bovenzi, A., Impellizzeri, M. F., Carminati, I., \& Castagna, C. (2013). Individual training-load and aerobic-fitness variables in premiership soccer players during the precompetitive season. The Journal of Strength \& Conditioning Research, 27(3), 631-636.

Rusko, H.K., Pulkkinen, A., Martinmäki, K., Saalasti, S., \& Kettunen, J. (2004). Influence of Increased Duration or Intensity on Training Load as evaluated by EPOC and TRIMPS. Medicine and Science in Sports and Exercise, 36(5) Supplement: S144.

Scott, B. R., Lockie, R. G, Knight, T. J., Clark, A. C., \& Janse de Jonge, X. A. K. (2013). A comparison of methods to quantify the inseason training load of professional soccer players. International Journal of Sports Physiology and Performance, 8(2), 195-202.

Seiler, K. S., \& Kjerland, G. Ø. (2006). Quantifying training intensity distribution in elite endurance athletes: is there evidence for an «optimal» distribution?. Scandinavian Journal of Medicine \& Science in Sports, 16(1), 49-56.

Seiler, S., Haugen, O., \& Kuffel, E. (2007). Autonomic recovery after exercise in trained athletes: intensity and duration effects. Medicine and science in sports and exercise, 39(8), 1366.

Stagno, K. M., Thatcher, R., \& Van Someren, K. A. (2007). Amodified TRIMP to quantify the in-season training load of team sport players. Journal of Sports Sciences, 25(6), 629-634.

Wallace, L. K., Slattery, K. M., \& Coutts, A. J. (2014).A comparison of methods for quantifying training load: relationships between modelled and actual training responses. European Journal of Applied Physiology, 114(1), 11-20.
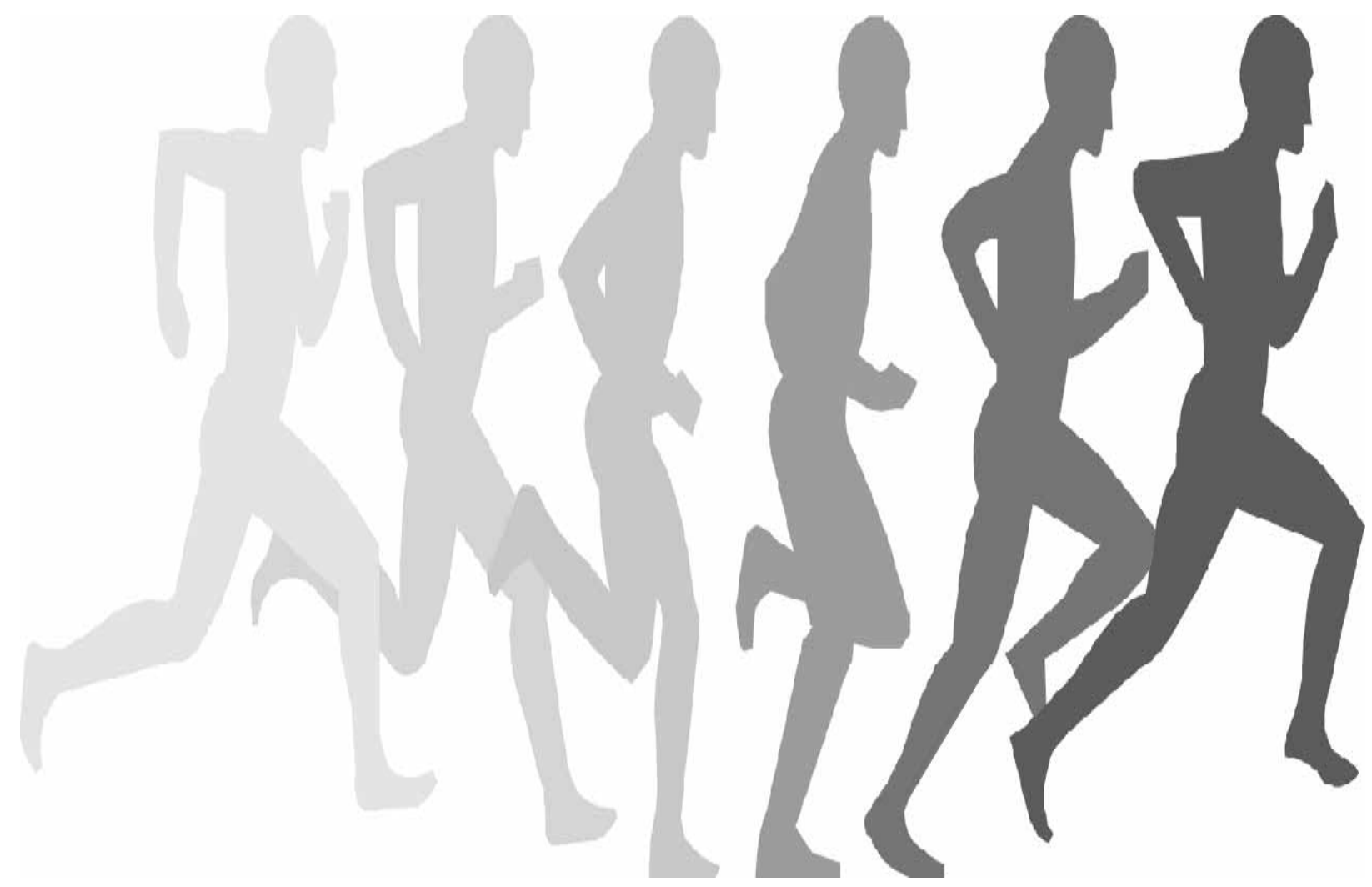